\title{
Increase in testosterone levels is related to a lower risk of conversion of prediabetes to manifest diabetes in prediabetic males
}

\author{
Michael Leutner · Caspar Matzhold · Luise Bellach · Evelyne Wohlschläger-Krenn · Robert Winker · Sonja Nistler • \\ Georg Endler · Stefan Thurner · Peter Klimek · Alexandra Kautzky-Willer (iD
}

Received: 25 September 2020 / Accepted: 27 May 2021 / Published online: 5 July 2021

(C) The Author(s) 2021

\begin{abstract}
Summary
Background Testosterone plays an important role in the regulation of glucose metabolism. While earlier studies have shown that it has a protective effect in males, unfavorable effects of testosterone on glucose metabolism have been reported in females; however, whether there is a sex-specific relationship between testosterone and glucose metabolism in patients with prediabetes has not been investigated in detail hitherto.

Methods This cross-sectional analysis investigated 423 males and 287 females with diagnosed prediabetes. Detailed assessment of their metabolic profiles was
\end{abstract}

The authors Michael Leutner and Caspar Matzhold
contributed equally to the manuscript.

M. Leutner · L. Bellach · A. Kautzky-Willer $(\bowtie)$

Department of Internal Medicine III, Clinical Division of Endocrinology and Metabolism, Unit of Gender

Medicine, Medical University of Vienna, Währinger

Gürtel 18-20, 1090 Vienna, Austria

alexandra.kautzky-willer@meduniwien.ac.at

C. Matzhold · S. Thurner · P. Klimek

Section for Science of Complex Systems, CeMSIIS, Medical University of Vienna, Spitalgasse 23, 1090 Vienna, Austria

C. Matzhold · S. Thurner · P. Klimek

Complexity Science Hub Vienna,

Josefstädter Straße 39, 1080 Vienna, Austria

E. Wohlschläger-Krenn $\cdot$ R. Winker $\cdot$ S. Nistler $\cdot$ G. Endler

Health and Prevention Center KFA,

Löblichgasse 14, 1090 Vienna, Austria

S. Thurner

Santa Fe Institute, 1399 Hyde Park Road, Santa Fe, NM

85701, USA

IIASA, Schlossplatz 1, 2361 Laxenburg, Austria

A. Kautzky-Willer

Gender Institute, 3571 Gars am Kamp, Austria performed, including a 2 -h oral glucose tolerance test (OGTT), HbAlc levels, calculation of insulin resistance with homeostatic model assessment for insulin resistance (HOMA-IR), assessment of lipid metabolism, anthropometric parameters and the fatty liver index (FLI). By using Spearman's correlation test, we investigated the sex-specific relationship between testosterone and metabolism in the prediabetic individuals. Results In the present study, prediabetic females (mean age 58.6 years, confidence interval [CI: 57.6y; $59.5 \mathrm{y}]$ ) were characterized by lower fasting plasma glucose levels (104.2 mg/dl [CI: $103.0 \mathrm{mg} / \mathrm{dl} ; 105.4 \mathrm{mg} /$ dl] vs. $106.9 \mathrm{mg} / \mathrm{dl}$ [CI: $106.0 \mathrm{mg} / \mathrm{dl} ; 107.8 \mathrm{mg} / \mathrm{dl}]$ ) and a lower FLI (49.5 [CI: 45.7; 53.2] vs. 58.8 [CI: $55.8 ; 61.8]$ ), but presented with a higher risk of developing manifest type 2 diabetes in the next 10 years (FINDRISK score: 17.6 [CI: 17.1; 18.1] vs. 16.1 [CI: 15.7; 16.5]) when compared to prediabetic males (mean age: 58.04 years [CI: $57.0 \mathrm{y} ; 59.1 \mathrm{y}]$ ). Testosterone was negatively related to insulin resistance (HOMA-IR: Spearman's $\rho$ : $-0.33, p<0.01), 2$-h stimulated glucose levels during the OGTT $(\rho=-0.18, p<0.01)$, HbAlc levels $(\rho=-0.13, p<0.05)$, FLI and BMI in prediabetic males; however, no relationship between testosterone and metabolic parameters could be found in prediabetic females.

Conclusion The increase of testosterone levels in males was related to a more favorable glucose metabolism, including lower HbAlc, lower stimulated glucose levels and higher insulin sensitivity; however, in prediabetic females, testosterone was not related to glucose metabolism.

Keywords Prediabetes - Testosterone - Insulin resistance $\cdot$ Fatty liver $\cdot$ Sex-specific differences 


\section{Introduction}

In recent years, the prevalence of type 2 diabetes mellitus (T2DM) has been steadily increasing and is predicted to rise even further worldwide by 2045 . Latest numbers from the International Diabetes Federation demonstrate that $9.3 \%$ of the world's population have T2DM [1]. Physiologically, sex hormones are involved in the regulation of glucose metabolism and are essential for maintenance of glucose homeostasis [2]. In men, testosterone is linked to insulin sensitivity and glycemic control [3]. Accordingly, in males there is a correlation between testosterone deficiency and the prevalence of prediabetes [4] and insulin resistance [5], meaning testosterone has favorable effects on glucose metabolism in males. Furthermore, low testosterone and sex hormone binding globulin (SHBG) levels in men can be linked to increased visceral fat content [6]. Conversely, higher testosterone levels and testosterone replacement therapy show beneficial effects on glucose homeostasis in men [7]; however, this does not seem to apply to women, since high testosterone blood levels in women are associated with T2DM [2, 3] and have been linked to diseases such as polycystic ovary syndrome [8]. Interestingly, there are sex-specific differences in the diagnosis of disturbances in glucose metabolism between men and women. Men more often suffer from increased fasting plasma glucose levels (FPG), whereas women more often suffer from impaired glucose tolerance, which is diagnosed with the oral glucose tolerance test (OGTT). The pathophysiological mechanisms behind these sex-specific differences are not entirely clear to date; however, one of the main reasons for the higher occurrence of increased FPG levels in men may be the elevated hepatic glucose output as well as impaired early insulin secretion. On the other hand, women more often suffer from impaired glucose tolerance (IGT) and an explanation for these sex-specific differences may be the slower duration of gastric emptying and intestinal glucose absorption in women [9]; however, especially the influence of sex hormones on disturbances of glucose metabolism in prediabetic patients is, overall, an unexplored area. Hence this study aimed to investigate the relationship between testosterone and glucose metabolism in prediabetic patients in a sex-specific manner.

\section{Methods}

\section{Study design and methods}

The cross-sectional study at hand (ethics approval from the Medical University of Vienna, 1089/2017) is a retrospective data analysis with an explorative character of first visits to the prediabetes clinic at "Health and Prevention Center KFA" in Vienna from October 2015 to September 2017. As part of a standard medical check-up, patients underwent a thorough physical examination, electrocardiogram, extensive blood analysis and assessment of vital parameters. Patients were classified as prediabetic patients according to the guidelines of the American Diabetes Association (ADA) if HbAlc was $\geq 5.7 \%$ and $<6.5 \%$ and/or if they had fasting blood glucose levels of $\geq 100 \mathrm{mg} / \mathrm{dl}$ and $<126 \mathrm{mg} / \mathrm{dl}$ and $/$ or $\geq 140 \mathrm{mg} / \mathrm{dl}$ and $<200 \mathrm{mg} / \mathrm{dl}$ in a 2 -h OGTT. All patients gave written informed consent. Patients suffering from chronic or acute infections, severe endocrine diseases (such as Cushing's disease), known hepatitis infection, HIV, present malignant diseases, systemic cortisone treatment, or testosterone substitution were excluded from the analysis.

\section{Calculations}

We calculated the homeostasis model assessment insulin resistance score (HOMA-IR) using basal insulin and glucose levels (HOMA-IR $=$ Ins $\times$ Gluc/405), the fatty liver index (FLI) and the Finnish diabetes risk score (FINDRISK score) $[10,11]$.

\section{Statistical analysis}

Descriptive data analysis was performed for all parameters. Continuous variables were summarized by mean and confidence intervals. Assumption of Gaussian distribution of parameters was decided by visual assessment of histograms and quantile-quantile plots (QQ plots). For normal distributed variables, a twosided t-test was used to assess differences between the two groups, and a Mann-Whitney U test was used for non-parametrically distributed variables. Due to the heterogeneous/skewed distribution of the individual variables, the Spearman correlation test was used for all groups to ensure reliable, comparable results. The statistical analysis was performed using the open source statistical analysis software R (version 4.01).

\section{Results}

\section{Baseline characteristics}

Table 1 shows the sex-specific comparison of the baseline characteristics between prediabetic men and women. In this analysis (f: 58.6 [CI: 57.6; 59.5] vs. m: 58.0 [CI: 57.0; 59.1] years), prediabetic males were characterized by lower total cholesterol and HDL cholesterol levels when compared to prediabetic females. The amount of visceral fat in the liver, measured with the fatty liver index, was significantly lower in prediabetic females.

However, while investigating glucose metabolism, we found a significantly higher FINDRISK score in prediabetic females, which means that they are at higher risk of developing manifest diabetes in the next 10 years. In general, prediabetic females presented with lower fasting plasma glucose (FPG); however, no 
Table 1 Baseline characteristics of prediabetic females and males

\begin{tabular}{|c|c|c|c|c|c|c|c|}
\hline \multirow[b]{2}{*}{ Variable } & \multicolumn{3}{|c|}{ Males } & \multicolumn{4}{|c|}{ Females } \\
\hline & N & Mean & $95 \% \mathrm{Cl}$ & $N$ & Mean & $95 \% \mathrm{Cl}$ & $p$-value \\
\hline Age (years) & 423 & 58.0 & $(57.0 ; 59.1)$ & 287 & 58.6 & $(57.6 ; 59.5)$ & 0.48 \\
\hline $\mathrm{BMI}\left(\mathrm{kg} / \mathrm{m}^{2}\right)$ & 419 & 28.2 & $(27.6 ; 28.7)$ & 284 & 28.3 & $(27.7 ; 28.9)$ & 0.87 \\
\hline Cholesterol (mg/dl) & 415 & 205.6 & $(201.8 ; 209.5)$ & 278 & 218.9 & $(214.4 ; 223.4)$ & $<0.01$ \\
\hline HDL cholesterol (mg/dl) & 416 & 56.2 & $(54.8 ; 57.7)$ & 279 & 65.9 & $(64.0 ; 67.8)$ & $<0.01$ \\
\hline LDL cholesterol (mg/dl) & 413 & 125.9 & $(122.4 ; 129.4)$ & 279 & 130.8 & $(126.6 ; 34.9)$ & 0.08 \\
\hline Triglycerides (mg/dl) & 416 & 115.7 & $(109.4 ; 121.9)$ & 279 & 110.2 & $(104.6 ; 115.8)$ & 0.88 \\
\hline Fatty liver Index & 294 & 58.8 & $(55.8 ; 61.8)$ & 197 & 49.5 & $(45.7 ; 53.2)$ & $<0.01$ \\
\hline Find risk score & 299 & 16.1 & $(15.7 ; 16.5)$ & 196 & 17.6 & $(17.1 ; 18.1)$ & $<0.01$ \\
\hline Glucose (mg/dl) & 402 & 106.9 & $(106.0 ; 107.8)$ & 267 & 104.2 & $(103.0 ; 105.4)$ & $<0.01$ \\
\hline Insulin $(\mu \mathrm{U} / \mathrm{ml})$ & 279 & 12.6 & $(11.6 ; 13.6)$ & 181 & 13.1 & $(12.1 ; 14.1)$ & 0.07 \\
\hline HOMA-IR & 197 & 3.5 & $(3.2 ; 3.8)$ & 134 & 3.4 & $(3.1 ; 3.8)$ & 0.84 \\
\hline Glucose levels (mg/dl)—OGTT $2 \mathrm{~h}$ & 289 & 117.4 & $(113.8 ; 121.0)$ & 196 & 119.8 & $(115.3 ; 124.3)$ & 0.41 \\
\hline Fasting glucose levels (mg/dl)—OGTT & 291 & 108.5 & $(107.5 ; 109.4)$ & 196 & 106.7 & $(105.4 ; 108.0)$ & 0.03 \\
\hline Testosterone (ng/ml) & 284 & 4.5 & $(4.3 ; 4.7)$ & 176 & 0.2 & $(0.2 ; 0.2)$ & $<0.01$ \\
\hline Vitamin D (ng/ml) & 404 & 27.2 & $(26.1-28.2)$ & 270 & 29.6 & $(28.1-31.2)$ & 0.02 \\
\hline $\mathrm{HbA} 1 \mathrm{c}>5.7 \%-\leq 6.5 \%$ & 131 & 5.9 & $(5.8 ; 5.9)$ & 134 & 5.9 & $(5.9 ; 5.9)$ & 0.22 \\
\hline Fasting glucose $100-<126 \mathrm{mg} / \mathrm{dl}$ & 252 & 109.2 & $(108.0 ; 110.0)$ & 147 & 109.0 & $(108.0 ; 110.1)$ & 0.53 \\
\hline Glucose levels $140-<200 \mathrm{mg} / \mathrm{dl}$ after $2 \mathrm{~h}$ during the $0 \mathrm{GTT}(\mathrm{mg} / \mathrm{dl})$ & 69 & 159.8 & $(156.2 ; 163.5)$ & 42 & 162.6 & $(157.6 ; 167.7)$ & 0.42 \\
\hline
\end{tabular}

$B M I$ body mass index, HDL high-density lipoprotein, LDL low-density lipoprotein, HOMA-IR homeostatic model assessment for insulin resistance, OGTT oral glucose tolerance test

Table 2 Sex-specific correlation analysis of testosterone with metabolic parameters

\begin{tabular}{|c|c|c|c|c|c|c|}
\hline \multirow[b]{2}{*}{ Variable } & \multicolumn{3}{|c|}{ Males } & \multicolumn{3}{|c|}{ Females } \\
\hline & $N$ & $\rho$ & $p$-value & $N$ & $\rho$ & $p$-value \\
\hline $\begin{array}{l}\text { Fasting glucose levels } \\
(\mathrm{mg} / \mathrm{dl})-0 \mathrm{GTT}\end{array}$ & 276 & -0.06 & 0.35 & 174 & -0.02 & 0.80 \\
\hline $\begin{array}{l}\text { Glucose levels } \\
(\mathrm{mg} / \mathrm{dl})-0 \mathrm{GTT} 2 \mathrm{~h}\end{array}$ & 274 & -0.18 & $<0.01$ & 174 & -0.05 & 0.53 \\
\hline HbA1c (\%) & 270 & -0.13 & 0.04 & 170 & 0.00 & 0.99 \\
\hline HOMA-IR & 195 & -0.33 & $<0.01$ & 131 & -0.08 & 0.38 \\
\hline Fatty liver index & 275 & -0.36 & $<0.01$ & 171 & 0.08 & 0.31 \\
\hline BMI $\left(\mathrm{kg} / \mathrm{m}^{2}\right)$ & 284 & -0.31 & $<0.01$ & 174 & 0.04 & 0.63 \\
\hline Cholesterol (mg/dl) & 282 & 0.03 & 0.61 & 171 & 0.04 & 0.62 \\
\hline HDL cholesterol (mg/dl) & 283 & 0.19 & $<0.01$ & 172 & -0.10 & 0.18 \\
\hline LDL cholesterol (mg/dl) & 281 & 0.07 & 0.28 & 172 & 0.05 & 0.52 \\
\hline Triglycerides (mg/dl) & 282 & -0.18 & $<0.01$ & 172 & -0.02 & 0.81 \\
\hline
\end{tabular}

sex-specific differences could be found for the stimulated glucose levels during the OGTT after $2 \mathrm{~h}, \mathrm{HbAlc}$ levels or the HOMA-IR.

\section{Correlation analyses of testosterone with metabolic parameters}

In a sex-specific correlation analysis (Table 2) our results show that there is a significant relationship between testosterone and glucose and lipid metabolism and between testosterone and anthropometric parameters in prediabetic males. The higher the levels of testosterone, the lower the levels of HbAlc and the lower the grade of insulin resistance measured with the HOMA index (Fig. 1) were in prediabetic males. Additionally, there was a negative relationship between testosterone and the stimulated glucose levels after $2 \mathrm{~h}$ in the OGTT in males (Fig. 1). We could also observe a favorable relationship between testosterone and the visceral fat content in the liver and anthropometric parameters (including FLI and BMI) in prediabetic males. Additionally, there was also a favorable relationship between testosterone and lipid parameters in prediabetic males, including a positive relationship with HDL cholesterol and a negative relationship with triglycerides; however, we could not find a relationship between testosterone and metabolic parameters in prediabetic females.

\section{Discussion}

The present study aimed to examine the sex-specific relationship between testosterone and glucose metabolism in prediabetic men and women. Prediabetic females were characterized by lower FPG levels and a lower FLI but had a higher risk of developing manifest diabetes in the next 10 years when compared to prediabetic men. Interestingly, testosterone seemed to have an overall favorable effect on metabolic profiles in prediabetic males, showing that there was a negative relationship with insulin resistance, HbAlc, stimulated glucose levels during the OGTT, FLI and BMI. Additionally, the increase in testosterone was related to a more favorable lipid profile in prediabetic men; however, in females we 
Fig. 1 Correlation between testosterone and a insulin resistance and b stimulated glucose levels in males. The linear relationship is indicated by the blue regression line with the corresponding standard error (gray error bar). The confidence lines for the predicted values are indicated by a red dotted line
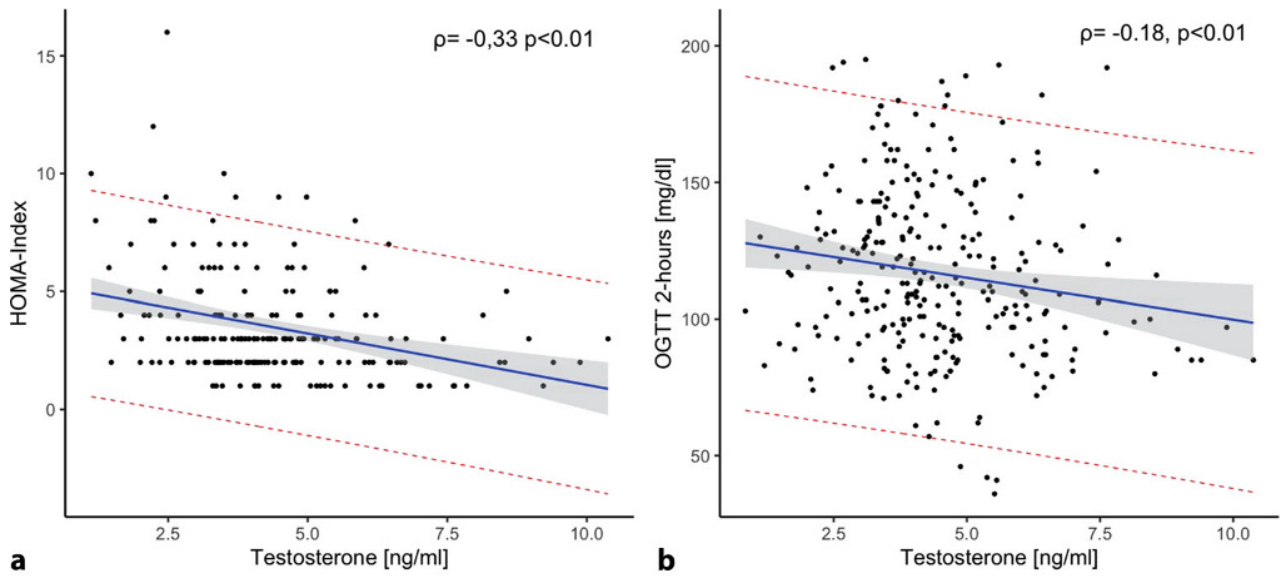

could not find a relationship between testosterone and metabolic parameters.

Although there is an abundance of available data showing an effect of testosterone on glucose metabolism in general or, more specifically, T2DM, there is only a limited amount of information on the influence of testosterone in the prediabetic state; however, existing data clearly indicate that prediabetic men show decreased testosterone or SHBG levels [12, 13]. In males, decreased testosterone levels are related to the development of disturbances in glucose metabolism, including increased risk of developing diabetes, and also showed a relationship with increased all-cause and cardiovascular mortality risk [6] as well as greater risk of developing a metabolic syndrome [14]. The significant role of testosterone in glucose metabolism in males is also reflected in the higher likelihood of lowered testosterone levels in T2DM patients compared to non-diabetics [15]. So far, it is not entirely known whether decreased levels of testosterone are the causal factor for metabolic derailment [9]. Nevertheless, one has to keep in mind that testosterone replacement therapy in hypogonadal diabetic males is related to an improvement of insulin sensitivity in patients with T2DM, metabolic syndrome or both [16]. Additionally, in patients receiving an androgen-lowering therapeutic regimen for the treatment of prostate cancer, the amount of visceral fat and insulin resistance increases under this therapy [15]. Furthermore, the detrimental effects androgen deficiency exerts on glucose homeostasis were also demonstrated in animal studies and cell culture experiments in which insulin secretion was impaired on top of testosterone deprivation [17]. Notably, there seems to be a bidirectional relationship between plasma testosterone and the integrity of glucose homeostasis. On the one hand, hyperglycemia suppresses gonadal testosterone production [18], while on the other hand testosterone deficiency leads to decreased muscle mass [19] and hence reduced glucose uptake in muscle [20]. Strikingly, in a prediabetic population, testosterone treatment showed a reduction in waist circumference and body weight, as well as an improvement in HbAlc and
FPG-in comparison, $40 \%$ of the prediabetic participants in the control group progressed to T2DM [21]. Interestingly, the effect of testosterone on the glucose metabolism seems to play a central role in the development of prediabetes. In a prospective clinical study, Minooee et al. detected that low testosterone levels were related to the development of prediabetes but could not find a relationship between the conversion of prediabetes to T2DM [22]. In the present study, we could show that especially in prediabetic men there is a significant relationship between testosterone and metabolic profiles. Increases in testosterone were related to a lower FLI, better insulin sensitivity, lower HbAlc and lower stimulated glucose levels, which are all markers directly related to the development of T2DM. One possible explanation for improved peripheral insulin sensitivity when testosterone levels are higher is the facilitation of glucose transporter type 4 (GLUT4) translocation to the myocytal membrane and, consequently, greater capacity for peripheral glucose uptake [20]. Furthermore, the protective effect testosterone exerts on peripheral glucose tolerance seems to be robust when correcting for age. Unlike in males, negative effects of testosterone on glucose metabolism have been reported in women [9]. For instance, elevated testosterone levels have been demonstrated in women with diabetes [23] - a constellation also typically present in polycystic ovary syndrome [24-26]. The role of testosterone in the prediabetic state in females is, overall, an unexplored area. Based on earlier results [9, 23], expectation of a negative effect of testosterone on the glucose metabolism in prediabetic females was high; however, in the present study we could not find a relationship between testosterone and insulin resistance, HbAlc, stimulated glucose levels during the OGTT or under fasting conditions and FLI or anthropometric parameters in prediabetic female subjects.

The role of testosterone in the sex-specific diagnostic differences in the disturbances of glucose metabolism is particularly interesting and our results provide more detailed knowledge on this topic. In general, males suffer more often from impaired 
fasting glucose levels driven by increased hepatic glucose output and dysfunctional early insulin secretion, whereas in females a higher occurrence of impaired glucose tolerance (IGT), mediated via peripheral insulin resistance, has been observed [9]. Interestingly, our results showing that there is a negative relationship between testosterone and stimulated glucose levels in prediabetic males demonstrate that testosterone could be an additional reason for the lower occurrence of IGT in males. As a possible mechanism, Navarro et al. showed that testosterone amplifies the effect of glucagon-like peptide 1 (GLP1) and enhances beta-cell function [27].

Finally, our results showing that testosterone seems to have favorable effects on the lipid profile in males are in line with earlier studies $[6,21]$.

Our study has limitations which have to be reported. Firstly, the present study has a cross-sectional study design and thus, we assessed correlations but not causal relationships. Secondly, we did not measure the levels of estrogen as well as the visceral fat content in the liver using gold standard methods such as magnetic resonance spectroscopy or ultrasound. Furthermore, we only assessed bivariate correlations between testosterone and metabolic parameters and therefore cannot rule out that the correlation in females is masked by a correlation with unobserved confounders; however, we noted that both males and females had comparable distributions of age and BMI. We had a smaller sample of females than males, which renders significant results in females less likely; however, males not only had lower $p$-values but also had slightly higher correlation coefficients.

In conclusion, our results show that there is a weak favorable relationship between testosterone and both glucose and lipid metabolism as well as with the visceral fat content in the liver, measured by FLI in prediabetic males. Interestingly, testosterone was not related to the metabolic profile in prediabetic females. Large prospective clinical studies are needed in order to investigate the sex-specific role of testosterone on metabolic profiles in more detail.

Funding Open access funding provided by Medical University of Vienna.

Conflict of interest M. Leutner, C. Matzhold, L. Bellach, E. Wohlschläger-Krenn, R. Winker, S. Nistler, G. Endler, S. Thurner, P. Klimek and A. Kautzky-Willer declare that they have no competing interests.

Open Access This article is licensed under a Creative Commons Attribution 4.0 International License, which permits use, sharing, adaptation, distribution and reproduction in any medium or format, as long as you give appropriate credit to the original author(s) and the source, provide a link to the Creative Commons licence, and indicate if changes were made. The images or other third party material in this article are included in the article's Creative Commons licence, unless indicated otherwise in a credit line to the material. If material is not included in the article's Creative Commons licence and your intended use is not permitted by statutory regulation or exceeds the permitted use, you will need to obtain permission directly from the copyright holder. To view a copy of this licence, visit http://creativecommons.org/licenses/by/4.0/.

\section{References}

1. Saeedi P, Petersohn I, Salpea P, Malanda B, Karuranga S, Unwin N, et al. Global and regional diabetes prevalence estimates for 2019 and projections for 2030 and 2045: results from the International Diabetes Federation Diabetes Atlas, 9th edition. Diabetes Res Clin Pract. 2019;157:107843.

2. Ding EL, Song Y, Malik VS, Liu S. Sex differences of endogenous sex hormones and risk of type 2 diabetes: a systematic review and meta-analysis. JAMA. 2006;295(11):1288-99.

3. LutzSZ, FritscheL, PeterA, RettigI, Wagner R, WillmannC, et al. Sex-specific associations of testosterone with metabolic traits. Front Endocrinol. 2019;10:90.

4. Arthur R, Rohrmann S, Møller H, Selvin E, Dobs AS, Kanarek N, et al. Pre-diabetes and serum sex steroid hormones among US men. Andrology. 2017;5(1):49-57.

5. Zitzmann M. Testosterone deficiency, insulin resistance and the metabolic syndrome. Nat Rev Endocrinol. 2009;5(12):673.

6. Rao PM, Kelly DM, Jones TH. Testosterone and insulin resistance in the metabolic syndrome and T2DM in men. NatRevEndocrinol. 2013;9(8):479-93.

7. Mohler ER III, Ellenberg SS, Lewis CE, Wenger NK, Budoff MJ, Lewis MR, et al. The effect of testosterone on cardiovascular biomarkers in the testosterone trials. J Clin Endocrinol Metab. 2017;103(2):681-8.

8. Ehrmann DA. Polycystic ovary syndrome. N Engl J Med. 2005;352(12):1223-36.

9. Kautzky-Willer A, Harreiter J, Pacini G. Sex and gender differences in risk, pathophysiology and complications of type 2 diabetes mellitus. Endocr Rev. 2016;37(3):278-316.

10. BedogniG, BellentaniS, Miglioli L, MasuttiF, PassalacquaM, Castiglione A, et al. The Fatty Liver Index: a simple and accurate predictor of hepatic steatosis in the general population. BMCGastroenterol. 2006;6:33.

11. Saaristo T, Peltonen M, Lindstrom J, Saarikoski L, Sundvall J, Eriksson JG, et al. Cross-sectional evaluation of the Finnish Diabetes Risk Score: a tool to identify undetected type 2 diabetes, abnormal glucose tolerance and metabolic syndrome. Diab Vasc Dis Res. 2005;2(2):67-72.

12. Boeri L, Capogrosso P, Pederzoli F, Ventimiglia E, Frego N, Chierigo F, et al. Unrecognized prediabetes is highly prevalent in men with erectile dysfunction-results from a cross-sectional study. J Sex Med. 2018;15(8):1117-24.

13. Ho CH, Yu HJ, Wang CY, Jaw FS, Hsieh JT, Liao WC, et al. Prediabetes is associated with an increased risk of testosterone deficiency, independent of obesity and metabolic syndrome. PLoS One. 2013;8(9):e74173.

14. Corona G, MonamiM, RastrelliG, AversaA, TishovaY,SaadF, et al. Testosterone and metabolic syndrome: a metaanalysis study. JSexMed. 2011;8(1):272-83.

15. Grossmann M. Testosterone and glucose metabolism in men: current concepts and controversies. J Endocrinol. 2014;220(3):R37-R55.

16. Jones TH, Arver S, Behre HM, Buvat J, Meuleman E, Moncada I, et al. Testosterone replacement in hypogonadal men with type 2 diabetes and/or metabolic syndrome (the TIMES2 study). Diabetes Care. 2011;34(4):828-37.

17. Mauvais-Jarvis F. Role of sex steroids in $\beta$ cell function, growth, and survival. Trends Endocrinol Metab. 2016;27(12):844-55. 
18. MareschCC, StuteDC,Alves MG, Oliveira PF, deKretserDM, Linn T. Diabetes-induced hyperglycemia impairs male reproductive function: a systematic review. Hum Reprod Update. 2018;24(1):86-105.

19. Kloner RA, Carson C 3rd, Dobs A, Kopecky S, Mohler ER 3rd. Testosterone and cardiovascular disease. J Am Coll Cardiol. 2016;67(5):545-57.

20. Tokarz VL, MacDonald PE, Klip A. The cell biology of systemic insulin function. J Cell Biol. 2018;217(7):2273-89.

21. Yassin A, Haider A, Haider KS, Caliber M, Doros G, Saad F, et al. Testosterone therapy in men with hypogonadism prevents progression from prediabetes to type 2 diabetes: eight-year data from a registry study. Diabetes Care. 2019;42(6):1104-11.

22. Minooee S, Ramezani Tehrani F, Rahmati M, Amanollahi Soudmand S, Tohidi M, Sabet Z, et al. The association between serum total testosteroneand progression of hyperglycemia: a 15-year prospective cohort study. Andrology. 2019;7(2):148-55.

23. Tramunt B, Smati S, Grandgeorge N, Lenfant F, Arnal JF, Montagner A, et al. Sex differences in metabolic regulation and diabetes susceptibility. Diabetologia. 2020;63(3):453-61.

24. Thong EP, Codner E, Laven JSE, Teede H. Diabetes: a metabolic and reproductive disorder in women. Lancet Diabetes Endocrinol. 2020;8(2):134-49.

25. Jayasena CN, Franks S. The management of patients with polycystic ovary syndrome. Nat Rev Endocrinol. 2014;10(10):624-36.

26. Moran LJ, Misso ML, Wild RA, Norman RJ. Impaired glucose tolerance, type 2 diabetes and metabolic syndrome in polycystic ovary syndrome: a systematic review and metaanalysis. Hum Reprod Update. 2010;16(4):347-63.

27. Navarro G, Xu W, Jacobson DA, Wicksteed B, Allard C, Zhang G, et al. Extranuclear actions of the androgen receptor enhance glucose-stimulated insulin secretion in the male. Cell Metab. 2016;23(5):837-51.

Publisher's Note Springer Nature remains neutral with regard to jurisdictional claims in published maps and institutional affiliations. 